\title{
Incorporación de la Ley de Gratuidad al Sistema de Educación Superior en Chile: la prolongación del subsidio a la demanda y la privatización del financiamiento público
}

Carlos Rodríguez-Garcés y Geraldo Padilla-Fuentes

\section{RESUMEN}

Utilizando datos oficiales, este artículo analiza los cambios que la nueva ley de Acceso Gratuito a la Educación Superior en Chile introdujo al financiamiento público, captación institucional y tasas de cobertura. Entre los resultados destaca la significativa inyección de recursos públicos pese al escaso nivel de cobertura alcanzado, una fuerte tendencia a su privatización y baja adhesión institucional. Con su implementación y gradualidad, esta ley acentúa la lógica del voucher, de acceso restringido y focalizado, mientras las instituciones, en un análisis de costo-beneficio, evalúan la captación de recursos y reducción de autonomía por regulación fiscal.

Palabras clave: educación superior, educación gratuita, financiamiento, privatización, política pública, Chile. 


\section{Incorporação da Lei de Gratuidade ao Sistema de Educação Superior no Chile: a prolongação} do subsidio à demanda e a privatização do financiamento público

\section{RESUMO}

Utilizando dados oficiais, este artigo analisa as mudanças que a nova lei de Acesso Gratuito à Educação Superior no Chile introduziu ao financiamento público, captação institucional e taxas de cobertura. Entre os resultados destaca a significativa injeção de recursos públicos pese ao escasso nível de cobertura alcançado, una forte tendência a sua privatização e baixa adesão institucional. Com sua implementação e gradualidade, esta lei acentua a lógica do voucher, de acesso restringido e focalizado, enquanto as instituições, em uma análise de custo-benefício, avaliam a captação de recursos e redução de autonomia por regulação fiscal.

Palavras chave: educação superior, educação gratuita, financiamento, privatização, política pública, Chile.

Incorporation of the Gratuity Law into the Higher Education System in Chile: the extension of the demand subsidy and the privatization of public financing

\section{ABSTRACT}

Making use of official information, this article analyzes the changes that the new law of Free Access to Higher Education in Chile introduced to public financing, institutional recruitment and coverage rates. Among the results, what stands out is the significant injection of public resources despite the low level of coverage achieved, a strong tendency towards privatization and low institutional adhesion. With its implementation and graduality, this law accentuates the logic of the voucher, of restricted and focused access, while the institutions, in a cost-benefit analysis, evaluate the attraction of resources and the reduction of autonomy through fiscal regulation.

Key words: higher education, free education, financing, privatization, public policy, Chile. 


\section{Introducción}

La educación en Chile transita por un dilatado periodo de discusiones y ajustes. La forma y sentido con que opera el sistema vigente se ha transformado en objeto frecuente de debate entre públicos tras haber funcionado alrededor de 30 años con escasas modificaciones (González, 2014). A este respecto, las protestas sociales y estudiantiles de 2006 marcaron un hito para la situación actual, y es que visibilizaron en la esfera pública la necesidad por mejorar la calidad y condiciones de acceso del sistema educativo. Eventos que junto a críticas por el cómo se orientaba, definía y evaluaba el sistema por criterios escasamente equitativos (Alarcón, Frites y Gajardo, 2015), acabaron por instalar en las agendas gubernamentales la urgencia por elevar los estándares de calidad desde una perspectiva multidimensional y mitigar la segregación socioeconómica entre establecimientos (Valenzuela, Bellei y De Los Ríos, 2010).

La respuesta más inmediata a este panorama de insatisfacción llegó en 2009 con la promulgación de la Ley 20370 o Ley General de Educación (LGE), que vino a derogar la Ley Orgánica Constitucional de Enseñanza (LOGE) dictada el último año de la dictadura que, además de no contar con legitimidad democrática, permitía, cuando no incentivaba, la selección escolar y participación de administradores privados sin regulación estricta, configurando lucro, segmentación y déficit de calidad como atributos adyacentes al sistema educativo. Ahora bien, aun cuando la LGE trató de poner atajo a ciertas irregularidades y crecimiento desmesurado del sector privado, fue débil en su gestión para controlar el lucro y segregación vinculada a las prácticas de selección que operaban en el sistema (Santa Cruz y Olmedo, 2012). Así mismo, prolongó la lógica de subsidio a la demanda vía voucher, característica del modelo de financiamiento chileno donde el Estado proporciona un subsidio al proveedor privado por cada alumno matriculado. Con todas sus debilidades y deficiencias, a la LGE se le reconoce haber inscrito la educación como un derecho social.

$\mathrm{Al}$ poco tiempo de promulgada la LGE, la tensión social y política se ubicó en los límites extremos del sistema educativo discutiendo, por un lado, sobre las oportunidades y características del sistema parvulario y, por el otro, las garantías de acceso y costos vinculados a la educación terciaria. En forma drástica, el Sistema de Educación Superior (SIES) se posicionó como eje de conflictos entre la ciudadanía y el deber del Estado por asegurar bienestar, en especial debido a denuncias por lucro, baja calidad, altos costos y percepción de discriminación en su funcionamiento. Así, el SIES debía confrontar algunos de sus aspectos que causaban malestar y ajustarse a las expectativas de una matrícula creciente, especialmente la que provenía de los quintiles de ingreso más bajos (Vergara, 2007), lo que suponía desvincularse de una lógica de subsidio a la demanda en un contexto donde la mercantilización y privatización, tal como acontecía en escuelas, colegios y liceos, eran prácticas extendidas en gran parte de la educación superior (Bellei, 2015).

Bajo este panorama se instala cada vez con mayor intensidad la urgencia por desarrollar una política de financiamiento que garantice acceso gratuito a la educación superior. Situación particularmente relevante en un contexto donde la educación tanto pública como privada es financiada por las familias, principalmente a través del endeudamiento. Por ello, el interés de este artículo es analizar los cambios que la política de gratuidad tuvo sobre el panorama financiero del SIES, especialmente, las modificaciones en la captación y distribución de fondos a instituciones públicas y privadas. Se busca contrastar el supuesto que, tal como está planteada la Ley de Gratuidad en Chile bajo el modelo de financiamiento imperante, ésta se constituye en un mecanismo que profundiza la lógica de subsidio a la demanda y privatiza el acceso a fondos públicos. 


\section{Contexto de la política de acceso gratuito a las instituciones de educación superior en Chile}

$\mathrm{Al}$ alero de la demanda social por mayores niveles de certificación y escasa regulación estatal, el panorama nacional en educación superior se ha configurado como una oferta formativa diversificada y masificada en que conviven instituciones públicas y privadas con heterogéneos niveles de calidad y criterios de admisión (Bravo y Manzi, 2002; García-Huidobro y Bellei, 2003; Donoso y Cancino, 2007; Castillo y Cabezas, 2010; Meller y Lara, 2010; Espinoza y González, 2011; Espinoza, 2013).

Si bien a través de los años el SIES ha buscado incrementar sus capacidades de preparación y certificación de capital humano, teniendo como misión aportar profesionales especializados y competentes al mercado laboral (Jiménez, 2009; OCDE, 2013), ello no ha sido gratuito. Sacar adelante una carrera profesional significaba para los estudiantes y sus familias asumir un importante compromiso económico, consecuencia directa de la lógica de financiamiento de las instituciones de educación superior (IES) legada por la LOCE y que establecía a la demanda como vía principal de captación de recursos. Según la OCDE, en 2009 Chile estaba entre los países miembros con mayor cargo privado por educación superior, donde un $85.4 \%$ era aportado por esta vía (OCDE, 2009), lo que equivalía, en el nivel de política pública y provisión del servicio, a conceptualizarla como bien de consumo cuyo acceso y más aún calidad podían estar sujetos a condiciones de negociación entre partes (Gajardo, 2014).

Todo esto, que para la sensibilidad social y política actual es indeseable, funciona sobre algunos principios de la teoría del capital humano y la correlación positiva que expone entre nivel educativo, tipo de empleo y niveles de renta (Smith, 1983; Brunner y Elacqua, 2003; García, 2005; Williamson, 2008; Beyer y Cox, 2011; Urzúa, 2012). La tradición económica fundamentó que los costos por acumular capital humano eran una inversión que producía altos retornos privados (Mincer, 1958; Brunner y Uribe, 2007), lo que vuelve legítimo para las IES cobrar por la prestación de sus servicios, debiendo operar como empresas eficientes, autofinanciadas y competitivas (Aedo y González, 2004). Asimismo, se postula que los costos de un financiamiento universal, además de ser escasamente viables en un panorama de presupuesto restringido, son altamente regresivos y sólo benefician privadamente a aquellos que acceden a este nivel educacional (Friedman y Friedman, 1980; Beyer y Cox, 2011). Huelga decir que los sistemas de créditos públicos y privados estaban ampliamente extendidos y se presentaban como una solución abierta a la comunidad cuyo pago podía pactarse racionalmente postitulación (The World Bank, 1988). Trasfondo económico que convertiría gradualmente la educación en una fábrica para la nueva economía del conocimiento (Herrera, 2013b).

Contrarios a esta perspectiva, diversos agentes exponen que mantener estos principios es un obstáculo a la equidad, fomenta una indeseable competencia y lógicas individualistas sobre el éxito como fruto exclusivo del esfuerzo, cuando no algo que se puede construir a costa de los otros, y permite funcionar al juego de oferta y demanda en un campo de derechos sociales fundamentales (Kremerman, 2013; Piscitelli, Adaime y Binder, 2010; Corvalán y García-Huidobro, 2015). Si el rol del Estado se reduce a alimentar el subsidio a la demanda, los mecanismos de financiamiento público se vuelven maleables y fáciles de caer en inconsistencias respecto de cautelar responsablemente un beneficio ciudadano (Atria y Sanhueza, 2013; Améstica, Gaete y Llinas, 2014). Considerando los beneficios sociales de la educación sobre el desarrollo económico, social y cultural común (Blanco, 2008; Ciclo de Diálogos sobre el Futuro de la Educación Chilena, 2014), según esta perspectiva se debiese dejar atrás un modelo de reproducción material de desigualdades y abrir paso al aseguramiento de derechos fundamentales (Zolezzi y 
De los Ríos, 2008; Corti, Oliva y De la Cruz, 2015), lo que significa por ejemplo disolver los mecanismos de crédito arancelario, los cuales han demostrado ser altamente rentables para la banca y un dispositivo de empobrecimiento para los egresados más vulnerables (Páez y Kremerman, 2016).

De todo esto, tres son los ejes de recurrente preocupación: educación como bien público, perjuicios del endeudamiento por créditos arancelarios en egresados y centralización del deber estatal en la provisión del derecho (Dias, 2008; Herrera, 2013a). Intentando cubrir los tres aspectos, en 2014 se iniciaron las conversaciones gubernamentales y parlamentarias para crear una Ley de Acceso Gratuito a las Instituciones de Educación Superior, intención que a finales de 2015 inició su trayectoria legislativa, primero como anexo al presupuesto del Ministerio de Educación (Mineduc) y actualmente como Ley 21 901, con el fin principal de asegurar financiamiento al costo de arancel y matrícula de los estudiantes. Si bien contemplaba en sus inicios cubrir solamente a estudiantes del sector universitario, se hizo extensible a todas las instituciones que conforman el SIES con independencia de su naturaleza pública o privada. La adhesión de estas últimas era voluntaria y según certificación de estándares de calidad, transparentes manejos financieros con reinversión de utilidades y adecuados criterios de admisión.

Respecto de los estudiantes beneficiarios, en lo inmediato se estableció como requisito el pertenecer a los primeros cinco deciles de menores ingresos en la población. Los altos costos que implicaba hacer frente al financiamiento de la educación superior con fondos públicos, no obstante la existencia de reformas tributarias para este efecto, derivó en hacer de la gratuidad un beneficio focalizado al que podía acceder sólo una parte de la población estudiantil.

De esta forma de implementación derivan dos conclusiones de relevancia. La primera de ellas es que contraviene el principio de universalidad que asiste a la educación en tanto derecho, cuya provisión debe ser garantizada, cuando no implementada, por el Estado. Esta restricción de acceso dualiza la matrícula al interior de una misma casa de estudios, distinguiendo entre alumnos con y sin gratuidad. En segundo lugar, profundizó las lógicas de subsidio a la demanda existentes en los otros niveles del sistema, ahora mediante la inauguración de un modelo de financiamiento mixto. En efecto, IES públicas y privadas continúan siendo de pago, con la diferencia que se inyecta una mayor cuantía de recursos fiscales que ahora, basado en los principios de no discriminación entre instituciones, pueden ser captados por entidades privadas. Así mismo, en la medida que los montos asignados por gratuidad están basados en el valor de un arancel de referencia, el diferencial resultante con el valor real cobrado por la casa de estudios debe ser cubierto por otras vías de financiamiento.

Pese a su implementación gradual, de acceso restringido y focalizado, la política de gratuidad mitigó relevantemente la carga económica familiar y su preocupación por sostener la formación educativa de los hijos/as. Desde la mirada institucional se convirtió en fuente significativa y estable de recursos con base en el perfil de matrícula atendida. Flujos económicos que, junto con acentuar la competencia, reconfiguraron las vías de financiamiento público y su tendencia a la privatización. Si previo a la política de gratuidad los créditos arancelarios y becas eran los medios privilegiados de asignación de fondos públicos vía estudiantil, ahora la gratuidad buscaría constituirse como pilar de sostenibilidad para las instituciones sin abandonar la lógica de subsidio a la demanda.

En consecuencia, resulta de interés para este artículo analizar los flujos y cobertura que tiene la política de gratuidad entre las distintas instituciones que integran el SIES, en razón de su tipología y adscripción; describir los atributos de la población beneficiaria; visualizar los ajustes de la oferta institucional con base a la captación y distribución del financiamiento público, además de explorar el nuevo abanico de 
estrategias de posicionamiento institucional según adhesión a la política. Uno de los supuestos principales busca exponer que la modalidad de implementación de la política de gratuidad de acceso restringido y no universal se aleja de la concepción de educación como derecho, extendiendo las lógicas de subsidio a la demanda con el riesgo añadido de profundizar la privatización de recursos públicos.

\section{Metodología \\ Instrumento}

Este artículo utiliza bases de datos de Contraloría General de la República (CGR), el Consejo Nacional de Educación (CNED) y la Comisión Nacional de Acreditación (CNA) correspondientes a la situación del año 2015, las que, con base en su naturaleza y fines, entregan información referente a matrícula, acreditación y financiamiento en el SIES. Con base en el código institucional, número que permite identificar unívocamente a la IES y que se encuentra presente en las distintas bases de datos, se realizó un único consolidado con las variables de interés.

Teniendo como unidad de agregación la institución educativa, se incorporó información sobre tipología de afiliación; estado y duración de acreditación; número de alumnos y participación en la matrícula; tipo, monto y captación de fondos públicos según vía institucional o estudiantil.

\section{Muestra}

La muestra global estuvo integrada por la totalidad de las instituciones de educación superior con base en su tipología y filiación, haciendo un total de 164 unidades de observación. En una segunda fase se extrajo de ellas una submuestra integrada exclusivamente por las 44 instituciones que se adscribían a la política de gratuidad en 2017, las cuales representan el $26.8 \%$ de la oferta institucional del SIES.

Tabla 1. Instituciones de educación superior en Chile, 2017

\begin{tabular}{|l|c|c|c|c|}
\hline \multirow{2}{*}{} & \multicolumn{2}{|c|}{ Muestra general } & \multicolumn{2}{c|}{ Submuestra gratuidad } \\
\cline { 2 - 5 } & $\boldsymbol{N}^{\circ}$ & $\%$ & $\mathbf{N}^{\circ}$ & $\%$ \\
\hline CFT & 60 & 36.6 & 6 & 13.6 \\
\hline IP & 43 & 26.2 & 6 & 13.6 \\
\hline U Estatal & 18 & 11.0 & 18 & 40.9 \\
\hline UP CRUCH & 9 & 5.5 & 9 & 11.4 \\
\hline UP NO CRUCH & 34 & 20.7 & 5 & 100.0 \\
\hline General & 164 & 100.0 & 44 & 20.5 \\
\hline
\end{tabular}

Nota: $N^{\circ}=$ valores absolutos; $C F T=$ Centro de Formación Técnica; $I P=$ Instituto Profesional; $U=$ Universidad; UP= Universidad Privada; $\mathrm{CRUCH}=$ Consejo de Rectores de las Universidades de Chile.

Fuente: elaboración propia según datos de Contraloría General de la República, Consejo Nacional de Educación y Comisión Nacional de Acreditación. 
De las 164 IES analizadas la mayor parte corresponde a instituciones de formación técnica de nivel superior (CFT) o profesional (IP), mientras un $37.2 \%$ son de carácter universitario. Representación que da cuenta de la heterogénea composición de la oferta educativa. A nivel universitario el mayor contingente refiere a instituciones privadas no adheridas al CRUCH (55.7\%).

Por su parte, la submuestra correspondiente a las 44 IES que adhieren en 2017 a la política de gratuidad, la mayor proporción corresponde a instituciones de carácter universitario $(72.8 \%)$, con una escasa participación de CFT (13.6\%), IP (13.6\%) y UP NO CRUCH (11.4\%).

\section{Procedimiento}

El análisis de los datos está compuesto por tres ejes. Primero se indaga sobre la matrícula y financiamiento fiscal que reciben las IES durante 2017 según naturaleza de su administración y carácter de afiliación, describiendo la concentración que alcanzan en cuanto a número de estudiantes y fondos públicos, hayan sido por becas y créditos, aportes institucionales o por gratuidad. Ello permite observar la ruta de distribución de los fondos públicos en un escenario de alta competencia donde los recursos siguen a los alumnos.

Como la política de gratuidad se aplica bajo la modalidad de aporte económico o beca, el segundo momento de análisis aborda el impacto que esta iniciativa tuvo en términos de cobertura y distribución del financiamiento según tipo de IES. Lo pretendido es visualizar cuáles son los ajustes que la gratuidad ha provocado en la configuración del financiamiento para instituciones, niveles actuales de matrícula beneficiada, captaciones y flujos.

\section{Resultados y análisis}

Las reformas iniciadas en los años ochenta al Sistema de Educación Superior (SIES) generaron una profunda transformación en el campo de la formación profesional, aumentando el número de instituciones y programas para atender a una población cada vez mayor y de heterogéneos perfiles socioeconómicos y escolares. En tal sentido, en 2017 había un total de 164 instituciones de educación superior (ES), con una matrícula total que bordea el millón doscientos cincuenta mil estudiantes (1 247 746), cifra que representa alrededor del 72\% de la cohorte poblacional entre 18 y 24 años proyectada según el Instituto Nacional de Estadísticas (INE). Explosivo crecimiento y alta cobertura en la matrícula con preminencia en la formación profesional de carácter universitario y de administración privada. En efecto, 6 de cada 10 estudiantes de ES (58.8\%) está matriculado en instituciones universitarias, y de éstos sólo un 26.6\% lo hizo en alguna entidad estatal.

Connatural al incremento en las tasas de matrícula, se instala la necesidad por resolver la problemática del financiamiento con participación del erario fiscal, en un panorama donde la educación comienza a concebirse como derecho con independencia relativa del carácter público o privado del oferente educativo. En tal sentido, el financiamiento fiscal realizado al SIES en 2017 se incrementó un 7.8\% respecto del año anterior, ascendiendo el gasto a MMUS\$ 3 076, de lo cual un 66.9\% fue captado por alguna de las 146 IES privadas, sean CFT (60), IP (43) o UES CRUCH (9) y NO CRUCH (34). Esta significativa privatización de los fondos públicos opera principalmente vía estudiantil, ya sea por otorgamiento de créditos, becas o gratuidad. 
Tabla 2. Matrícula y financiamiento al SIES según tipo de IES

\begin{tabular}{|l|c|c|c|c|c|c|c|}
\hline & & \multicolumn{2}{|c|}{ Matrícula total } & \multicolumn{4}{c|}{ \% Captación FP } \\
\cline { 2 - 8 } & IES & $\mathbf{N}^{\circ}$ & $\%$ & Ins & Byc & Grat & Total \\
\hline CFT & 60 & 136,789 & 10.9 & 2.6 & 6.4 & 8.2 & 6.0 \\
\hline IP & 43 & 377,354 & 30.2 & 0.8 & 17.3 & 17.2 & 12.8 \\
\hline U Estatal & 18 & 194,841 & 15.6 & 55.6 & 15.0 & 35.9 & 33.1 \\
\hline UP CRUCH & 9 & 155,752 & 12.5 & 37.3 & 14.8 & 25.8 & 24.6 \\
\hline UP NO CRUCH & 34 & 383,010 & 30.7 & 3.7 & 46.5 & 12.9 & 23.6 \\
\hline Total & 164 & $1,247,746$ & 100.0 & 100.0 & 100.0 & 100.0 & 100.0 \\
\hline
\end{tabular}

Nota: $C F T=$ Centro de Formación Técnica; $I P=$ Instituto Profesional; $U=$ Universidad; $C R U C H=$ Consejo de Rectores de las Universidades de Chile; IES = Instituciones de Educación Superior; $N^{\circ}=$ Número de estudiantes; $F P=$ Financiamiento Público; Ins = vía Institucional; ByC= vía Becas y Créditos; Grat= vía Gratuidad. Base IES sobre total de instituciones reconocidas oficialmente, excluidas FFAA.

Fuente: elaboración propia según datos Contraloría General de la República, Consejo Nacional de Educación y Comisión Nacional de Acreditación.

A la presencia de un sistema de educación superior que se expande en términos de cobertura con una matrícula eminentemente universitaria y de carácter privado, se suma como fenómeno reciente el constante incremento en la captación de los fondos públicos por parte de entidades privadas. Las IES del Estado, con las cuales éste comparte misma naturaleza y objetivo, captan un escaso $33.1 \%$ del total de financiamiento, porcentaje que en la última década ha experimentado un descenso preocupante. En una política de voucher donde los fondos siguen a los alumnos, el financiamiento público fluye cada vez con mayor intensidad hacia instituciones de naturaleza privada, tendencia que se vio acentuada con la entrada en vigencia de la política de gratuidad.

Conforme se diversifica y segmenta la cobertura y matrícula con base en heterogéneos perfiles socioeducativos, se tensiona la modalidad de financiamiento vinculada al endeudamiento familiar y acceso al crédito bancario (Rodríguez, 2012;
Guerrero y Campbell, 2016), presionando hacia una mayor participación por parte del Estado en materia educativa. En efecto, el ordenamiento jurídico y constitucional chileno define al Estado en un rol meramente subsidiario en la provisión y administración de servicios sociales, reconociendo y amparando al conjunto de entidades privadas que se estructuran y organizan en la sociedad para tales efectos, a las cuales garantiza adecuada autonomía.

La entrada en vigencia de la ley de gratuidad no pone en cuestión este principio, sino más bien da continuidad y acentúa el modelo transferencista y subsidiario, ahora en un contexto de mayor contribución financiera y sin distingo del carácter y naturaleza de la institución. En efecto, la mayor asignación de recursos posibilita ampliar el número de becas y créditos estudiantiles a la generalidad del sistema; situación que es coronada con la implementación de la Ley de gratuidad. En este contexto, la inyección de recursos fiscales a las IES vía aporte estudiantil 
adquiere relevancia con independencia del carácter público o privado de la institución, constituyéndose en una modalidad de financiamiento compartida, por lo que a la otrora competencia por la obtención de matrícula se añade la disputa por los fondos públicos como forma de financiamiento, en un escenario caracterizado por fuertes asimetrías. Por cada 10 pesos que el Estado invierte en el SIES 7 de ellos $(73 \%)$ son por vía Estudiantil, y el $75.3 \%$ de éstos son captados por instituciones privadas.

En tal escenario, y al otorgar fondos públicos para financiar arancel y matrícula con independencia relativa del carácter de la IES, la Ley de Acceso Gratuito a las Instituciones de Educación Superior prolonga las lógicas de subsidio a la demanda que han caracterizado el modelo de financiamiento y delimitado la responsabilidad y rol del Estado en esta materia.

En la actualidad tan sólo una de cada cuatro instituciones que conforman el SIES adhiere a la Ley de gratuidad, representando en términos de matrícula tan sólo un 52.4\% del alumnado. Baja adscripción que además está segmentada por tipología de IES. En el sector universitario sólo un 33.5\% de la matrícula tiene opciones de acceder a gratuidad, observándose una fuerte heterogeneidad en razón del carácter de la entidad. Para el caso de las universidades que no pertenecen al Consejo de Rectores (34), y que en conjunto aglutinan el 30.7\% de la matrícula, solo cinco de ellas están incluidas en el marco de gratuidad, lo que representa un $17.7 \%$ de su conglomerado estudiantil.

Por su parte, la formación técnico profesional sigue un comportamiento peculiar. De los 60 CFT que integran el SIES y que reúnen el 10.9\% de la matrícula general, solo seis de ellos adhiere a gratuidad; baja adhesión institucional que no impacta negativamente en el volumen de potenciales beneficiarios que bordea el $48.7 \%$. Este comportamiento de baja adscripción institucional pero alta cobertura proyectada puede explicarse por las diferenciadas y segmentadas características que tiene el estamento técnico profesional en cuanto a presencia territorial y captación de matrícula. Así por ejemplo, el Instituto Nacional de Capacitación (INAGAP), mega centro de la formación técnico profesional, concentra por sí solo el 34.9\% de la matrícula de este segmento, y alcanza una participación del 74\% respecto de los CFT que adhieren a gratuidad.

Cabe recordar que la decisión de participar en el marco de gratuidad opera en un contexto de voluntariedad, expresión de autonomía decisional que puede derivar en autoexclusión si la evaluación de intereses de política institucional así lo define. A ello se suma un conjunto de requisitos y condiciones que las IES que desean adherir deben cumplir, referidas a la acreditación institucional, disponer de una vía de admisión transparente y objetiva, así como declarar dentro de su misión la no consecución de fines de lucro.

Con base a la adhesión institucional observada (44 IES), la gratuidad como política de financiamiento en la educación superior presenta un significativo déficit en su cobertura por matrícula disponible, donde uno de cada dos alumnos $(47.6 \%)$ asiste a IES no adscritas. Del total de estudiantes matriculados en instituciones adscritas ( $\mathrm{n}=588,697)$, un $44.5 \%$ fueron beneficiados en 2017, aunque considerando la totalidad de los estudiantes que integran el SIES las tasas de cobertura real alcanzaron un exiguo $23.3 \%$. 
Tabla 3. Matrícula gratuidad según IES adscritas

\begin{tabular}{|c|c|c|c|c|c|}
\hline & \multicolumn{2}{|c|}{ Adhesión } & \multicolumn{2}{|c|}{ Gratuidad IES adscritas } & \multirow[b]{2}{*}{$\begin{array}{c}\text { Cobertura } \\
\text { real }\end{array}$} \\
\hline & $N^{\circ}$ IES & $\%$ alumnado* & Captación & Cobertura & \\
\hline CFT & 6 & 48.7 & 12.0 & 48.8 & 23.8 \\
\hline IP & 6 & 43.3 & 23.7 & 41.9 & 18.1 \\
\hline u Estatal & 18 & 100.0 & 31.9 & 47.0 & 47.0 \\
\hline UP CRUCH & 9 & 100.0 & 21.7 & 40.4 & 40.4 \\
\hline UP NO CRUCH & 5 & 17.7 & 10.7 & 48.2 & 8.5 \\
\hline Total & 44 & 52.4 & 100.0 & 44.5 & 23.3 \\
\hline
\end{tabular}

Nota: * =valor de referencia solo matrícula presencial y pregrado $(n=1.122 .541) ; C F T=$ Centro de Formación Técnica; IP= Instituto Profesional; U = Universidad; $\mathrm{CRUCH}=$ Consejo de Rectores de las Universidades de Chile; IES=Instituciones de Educación Superior. Fuente: elaboración propia según datos Contraloría General de la República, Consejo Nacional de Educación y Comisión Nacional de Acreditación.

En tal panorama, aproximadamente tres de cada cuatro estudiantes $(76.7 \%)$ no se constituye en población objetivo, sea porque no cumple con los requisitos de perfil socioeconómico o bien está matriculado en una institución no adscrita. Alto déficit de cobertura que cuestiona la política de gratuidad como medida efectiva de financiamiento, por cuanto un significativo contingente del estudiantado debe asumir los altos costos derivados de la formación con cargo privado, sea mediante el presupuesto familiar o el endeudamiento. Esta situación se hace particularmente compleja para estudiantes de institutos profesionales o centros de formación técnica, estamentos caracterizados por el vulnerable perfil socioeconómico de su alumnado (Larrañaga, Cabezas y Dussaillant, 2013), donde las coberturas reales alcanzan un $18.1 \%$ y $23.8 \%$ respectivamente.

Si bien en términos de distribución la mayor proporción de quienes son beneficiarios corresponde al ámbito universitario (64.3\%), particularmente matriculados en entidades de administración estatal $(31.9 \%)$, esto es resultado, por un lado, de la conformación desigual del SIES cuya matrícula tiende a concentrarse en el espacio universitario; y por otro, de los diferenciados niveles de adhesión, especialmente bajos para el caso de las UP NO CRUCH. Por ello, al considerar los porcentajes de incidencia al interior de las instituciones inscritas con base a su tipología, las coberturas tienden a la homogeneidad, con rangos que transitan entre un $40.4 \%$ en el caso de las UP CRUCH y $48.8 \%$ en los CFT.

Dado que en la práctica la política de gratuidad actúa como subsidio a la demanda asignado en función de vulnerabilidad socioeconómica, y que el sistema de educación superior en Chile cuenta con persistentes niveles de segmentación social, la extendida homogeneidad en las tasas de cobertura institucional resulta peculiar, más aún si consideramos la tendencia histórica de las clases baja y media baja por ingresar a centros educativos orientados al 
mercado laboral (CFT e IP) o con flexibles criterios de admisión (universidades no GRUCH). En efecto, atendiendo a los criterios de focalización y segmentación, era razonable esperar una distribución de fondos vía gratuidad más diferenciada a la que reportan los datos; situación que podría responder a las siguientes ideas o hipótesis. Primero, la similitud entre tasas de cobertura puede ser efecto de un eventual sesgo de selección que opera en la base decisional de un sistema socialmente estratificado donde las IES buscan maximizar la relación costo beneficio. Para el caso de las universidades privadas, la decisión institucional de adscribir a la política de gratuidad se tomaría en razón de la conformación socioeconómica de su matrícula. De este modo, las no adscritas privilegiarían la mantención del financiamiento privado dada la mayor capacidad de pago de su alumnado.

Otro argumento, más controversial, es que la homogeneidad de coberturas se realiza con la intencionalidad política de mantener los equilibrios entre instituciones en lo que a financiamiento público refiere, basado en el principio de no discriminación por naturaleza o carácter público/privado. Así, la gratuidad se constituye en una forma de financiamiento estatal por vía estudiantil de implementación gradual y homogénea entre sectores, que no dista significativamente del formato de becas aun alcanzando la anhelada universalidad. En lugar de potenciar una política de financiamiento basal hacia las instituciones de vocación pública, se prolonga la lógica del subsidio a la demanda donde los fondos públicos siguen a unos alumnos cada vez más atraídos por la oferta privada.

Sobre las condiciones de adjudicación para el estudiante, los requisitos con mayor capacidad de discriminación son estar matriculado en una institución elegible por la ley y cumplir con el atributo socioeconómico de provenir de algún hogar ubicado en los primeros cinco deciles de ingresos. Como en primera instancia el financiamiento por gratuidad se consideró ítem fijo en los gastos del Ministerio de Educación en 2016, la selección fue efecto ineludible de una racionalidad de focalización en un contexto de estrechez presupuestaria. No obstante, de no mediar esta gestión de recursos limitados con atención preferencial hacia los grupos más vulnerables haciendo extensivo el acceso universal, igualmente las tasas de cobertura potencial bordearían tan sólo el 52\%.

En 2017, uno de cada dos alumnos estaba matriculado en alguna IES que no adhiere o no es elegible por ley de gratuidad (ver tabla 3), sea por no disponer de un sistema de selección de su matrícula objetivo y transparente, perseguir fines de lucro o no dar garantías sobre la calidad de sus procesos formativos. Respecto de esta última condición, si bien los datos del Consejo Nacional de Acreditación (2017) informan un alto porcentaje de instituciones no acreditadas, que para el caso de los CFT alcanza el $60 \%$, un $55 \%$ de los IP y un $34.3 \%$ de las UP, su impacto en términos de alumnado es reducido, por cuanto al considerar su matrícula concentran respectivamente el $14 \%, 10.5 \%$ y $22.4 \%$ dentro de su segmento, lo que en términos globales representa un escaso $12.3 \%$ de la población de pregrado en educación superior. Cabe hacer presente que la acreditación institucional es un sistema de certificación que un organismo externo realiza respecto de la calidad de la formación de pregrado y que condiciona el acceso a fondos públicos. Credencial que al ser voluntaria, incentiva por un lado la participación en este proceso evaluativo por parte de quienes concentran mayor tasa de matrícula como vía para acceder a financiamiento fiscal; por otro, margina a gran parte de las instituciones que no cumplen con este estándar de calidad, que si bien atienden a los segmentos más vulnerables, son las de menor concentración de matrícula.

El segundo requisito, poseer un sistema de admisión objetivo, transparente y pertinente que no implique discriminación arbitraria, condiciona con mayor intensidad la incorporación a ley de gratuidad. La 
educación superior en Chile se caracteriza por presentar una modalidad dual de admisión. Por un lado, el Sistema Único de Admisión (SUA), al cual adhiere la mayor parte de las universidades, sirve como vía de ingreso para sólo un 37.9\% del alumnado, mientras el grueso de las instituciones utiliza mecanismos autónomos de admisión caracterizados por su escaso rigor, opacidad y heterogeneidad. Consensuar entre las instituciones un sistema único de admisión y de similares atributos al SUA se constituye en uno de los principales desafios para la implementación de la política de gratuidad, no sólo por los recursos económicos y logísticos que implica su diseño sino también por la resistencia a la pérdida de autonomía. Por lo demás, procedimientos selectivos, en particular por perfil académico o trayectoria escolar, derivarían en la pérdida de una significativa porción de la matrícula que otrora sólo requería la licencia de enseñanza media para inscribirse en algún programa, arriesgando por extensión la viabilidad económica de la institución, toda vez que el sistema de admisión vía pruebas de admisión suele ser una barrera difícil de sortear (Valdivieso, 2006; Contreras, Corbalán y Redondo, 2007). A modo de ejemplo, en el proceso de admisión 2017 un 30.5\% de los estudiantes que rindieron las Pruebas de Selección Universitaria no superó los 450 puntos, cifra engrosada principalmente por los estudiantes más vulnerables. El sesgo de selección que se le atribuye al SUA, fuertemente alineado con atributos socioeconómicos, es una debilidad recurrentemente informada por el mundo académico, y se constituye en argumento para rechazar su aplicación por parte de instituciones privadas al constituirse en un mecanismo que legitima desigualdades sociales de origen (Corporación de Universidades Privadas de Chile, 2016).

El tercer eje que cruza por el debate sobre cobertura de gratuidad se asocia con el sensible ejercicio del lucro en la educación. Si bien la Ley Orgánica Constitucional de Enseñanza (LOGE) que reformó el SIES en Chile establecía, con excepción de los CFT e IP, que toda universidad privada no podía tener fines de lucro (LOCE, 1990), esta restricción tempranamente derivó en un conjunto de estrategias fraudulentas para la captación de utilidades por parte de las entidades controladoras (Educación 2020, 2018). Vacío y desregulación que buscó corregirse recientemente con la Ley 21091 (2018) sobre Educación Superior promulgada en 2018, donde se establece que cualquier IES que pretenda reconocimiento oficial y acceso a financiamiento fiscal debe cumplir una serie de condiciones vinculadas al no lucro y transparencia en el uso de activos generados con ocasión del servicio educativo. Por tanto, no estar adscrita a política de gratuidad es también una decisión políticoadministrativa de instituciones que, incumpliendo aspectos éticos y legales referentes a la reinversión de fondos, evitan la sobre exposición de sus movimientos presupuestarios.

En consecuencia, las restricciones institucionales referentes a acreditación, admisión y lucro actuarían dinámicamente y según la particularidad de cada institución, las cuales definen su estrategia de posicionamiento en un continuo paradigmático que transita entre la educación como derecho social y su concepción como bien de consumo, situación que explica la baja adhesión institucional $(26.8 \%)$ y cobertura real $(23.3 \%)$. Pese a esto, la política de gratuidad representa una fuerte responsabilidad económica para el gasto público, acaparando más de un tercio de lo asignado al sistema de educación superior (33.8\%).

El aporte que el Estado realiza a las IES, que en 2017 correspondió al 20.1\% del presupuesto disponible para el Ministerio de Educación, opera bajo las modalidades de financiamiento institucional y financiamiento estudiantil (Marcel y Tokman, 2005; Donoso, 2002; Rodríguez y Padilla, 2017). Como expone la tabla 3, la vía estudiantil correspondiente a becas, créditos y glosa de gratuidad, se ha convertido en la columna vertebral del financiamiento público a las IES $(73 \%)$, con una pronunciada tendencia a su captación por entidades privadas. En efecto, 
instalada la industria educativa masiva, privada y segmentada, la privatización de fondos públicos vía estudiantil emerge como atributo distintivo de un modelo de financiamiento de subsidio a la demanda, donde a los tradicionales créditos y becas se añade la política de gratuidad. Modalidad de financiamiento público que, al no operar con las restricciones del aporte institucional, estaría abierta a la competencia entre IES con independencia de su administración pública o privada.

Tabla 4. Financiamiento público y gratuidad

\begin{tabular}{|l|c|c|c|c|c|}
\hline \multirow{2}{*}{} & SIES & \multicolumn{2}{|c|}{ F. Estudiantil (\%) } & \multicolumn{2}{c|}{ F. Gratuidad (\%) } \\
\cline { 2 - 6 } & MMS & Comp & Cap & Comp & Cap \\
\hline CFT & 119,036 & 88.2 & 7.2 & 46.2 & 8.2 \\
\hline IP & 255,214 & 98.4 & 17.2 & 45.4 & 17.2 \\
\hline U Estatal & 659,448 & 54.5 & 24.7 & 36.7 & 35.9 \\
\hline UP CRUCH & 491,093 & 59.1 & 19.9 & 35.5 & 25.8 \\
\hline UP NO CRUCH & 470,288 & 95.7 & 30.9 & 18.5 & 12.9 \\
\hline Total & $1,995,079$ & 73.0 & 100.0 & 33.8 & 100.0 \\
\hline
\end{tabular}

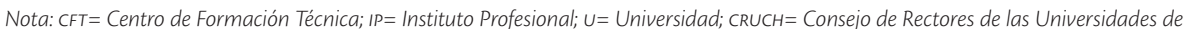
Chile; IES = Instituciones de Educación Superior; F= Financiamiento Público. Fuente: elaboración propia según datos Contraloría General de la República, Consejo Nacional de Educación y Comisión Nacional de Acreditación.

En términos generales, y tal como se observa en la tabla 4, la implementación política de gratuidad representó, por un lado, una significativa asignación de fondos presupuestarios y, por otro, una importante tendencia de captación por parte de entidades privadas. En efecto, el 33.8\% del financiamiento público al SIES y un $6.8 \%$ del presupuesto global que dispuso Mineduc en 2017 fueron comprometidos por esta vía, constituyendo la piedra angular en la asignación de recursos estatales para el financiamiento de arancel y matrícula, particularmente en IES privadas. A este respecto los datos son ilustrativos, el 55.3\% del financiamiento estudiantil es captado por instituciones privadas no tradicionales, y en lo que refiere a gratuidad alcanzan un $38.3 \%$.
Los datos reportan altos costos asociados a la política de gratuidad en comparación a los bajos índices de cobertura real que alcanza, lo cual pone en entredicho el ideario político de alcanzar su universalidad, de no mediar significativas y estructurales reformas presupuestarias y/o tributarias con el fin de dar viabilidad económica al proyecto. Problemática que se complejiza en atención del incremento en los costos de arancel que supone la sofisticación de planes y programas en un contexto de mejoramiento continuo de la calidad, y el aumento natural de la matrícula producto del incentivo que la misma gratuidad genera en la población, particularmente en segmentos con menor tasa de cobertura. Por otra parte, actualmente esta política solo financia la 
duración nominal de los programas, no haciéndose cargo del rezago académico, especialmente acentuado en el contexto universitario, dejando a la deriva un contingente significativo de estudiantes que, al expirar el beneficio, ven complicada la finalización de sus estudios.

\section{Conclusiones}

La promulgación de la Ley de Acceso Gratuito a las Instituciones de Educación Superior fue el producto de una demanda arrastrada por años y expresión de múltiples intereses ciudadanos y políticos. En su ideario buscaba posicionar a la educación como un derecho social de carácter universal con garantía estatal, aunque la gradualidad de su implementación implicó en la práctica prolongar el subsidio a la demanda disminuyendo, en una lógica no muy diferente a la de becas, los costos monetarios de arancel y matrícula derivados del acceso a la educación superior por parte de los quintiles más pobres.

En su puesta en marcha, la política de gratuidad derivó en una asignación gradual de recursos públicos y una creciente tendencia a su privatización como expresión de las particularidades de un sistema de educación superior masificado, diversificado y con fuerte participación de oferentes privados. Así también, los altos costos que implicaba para el erario público forzaron al ajuste de expectativas desde la universalidad hacia un beneficio de acceso limitado y focalizado.

Los datos analizados en torno a la implementación de la ley de gratuidad observan una baja adhesión institucional y escasa cobertura real de beneficiados, a pesar de la importante inyección de recursos realizada. Adicionalmente, se constata una significativa captación de fondos públicos por IES privadas a través de los aportes estudiantiles, asignaciones fiscales que no presentan las restricciones del aporte institucional fiscal. Esta tendencia, si bien ha sido ya observada por otros estudios, se profundiza por la irrupción de esta ley y su implementación bajo la lógica voucher.
Ahora bien, aun cuando esto resuelve la carga económica en estudiantes y familias pertenecientes al 50\% más pobre de la población que antes recurrían al endeudamiento crediticio para financiar arancel y matrícula, la modalidad de implementación tipo beca cuestiona la concepción de educación como derecho social para todas y todos. $\mathrm{Y}$ es que en términos prácticos y en la medida que su extensión de cobertura está determinada por presupuesto fiscal y sensibilidad ideológica del gobierno de turno, la política de gratuidad corre el riesgo de solidificarse en la batería de becas, es decir, constituirse en un beneficio restringido al cual se accede por condición socioeconómica.

Por otra parte, su operacionalización no logra desprenderse de la racionalidad de subsidiar la demanda, concepción que estructuralmente ha definido la política de financiamiento educativo en Chile. En efecto, es el alumno el que se hace acreedor del beneficio de gratuidad, el cual puede hacer efectivo al matricularse en cualquier IES adherida, decisión fuertemente influenciada por la propaganda y mecanismos especulativos diseñados por las instituciones para captar matrícula, sin que ello signifique necesariamente estar considerando estándares de calidad y/o excelencia.

Que a los fondos de gratuidad se acceda a través del perfil del alumnado es una característica sustancial para comprender los posicionamientos institucionales, particularmente en el caso de corporaciones privadas que, cumpliendo con los criterios de base, definen su adhesión en un espacio evaluativo que relaciona por un lado el allegamiento de recursos públicos que la política de gratuidad provee y por otro el nivel de injerencia sobre la gestión financiera y administrativa que ella condiciona.

Lo anterior hace cuestionar la factibilidad de alcanzar la universalidad proyectada en el mediano plazo, incorporando además un escollo ético-político aún no del todo resuelto acerca de la responsabilidad estatal de distribuir dineros públicos a un conjunto 
de instituciones fuertemente diferenciadas en términos de calidad, compromiso social y transparencia financiera. Aquí planteamos que la solución a éste y otros problemas del SIES no se traduce en reasignaciones, ajustes o incrementos al presupuesto para financiar los gastos educativos de una matrícula en permanente crecimiento, o bien a proscribir a las IES privadas de este beneficio, marginando de paso a un importante volumen de estudiantes y que tienden a ser precisamente los más vulnerables. Más bien, el debate debería centrarse en cómo instalar un sistema de financiamiento basal eficiente que brinde estabilidad al desarrollo de los proyectos educativos en instituciones con vocación pública, con las cuales el Estado comparte la responsabilidad de proveer servicios asociados a derechos. Esto es, un servicio de calidad, exento de lucro en su provisión y universalmente gratuito.

\section{Referencias}

Aedo, G. y L. González (2004), "La educación superior en Chile", Calidad en la Educación, núm. 21, pp. 61-85.

Alarcón, J., C. Frites y C. Gajardo (2015), "El devenir de la utopía economicista en el sistema escolar Chileno", Educação e Pesquisa, vol. 41, núm. 4, pp. 1-13.

Améstica, L., H. Gaete yJ. Llinas (2014), "Segmentación y clasificación de las universidades en Chile: desventajas de inicio y efectos de las políticas públicas de financiamiento", Revista Chilena de ingeniería, vol. 22, núm. 3, pp. 384-397.

Atria, F. y C. Sanhueza (2013), "Propuesta de gratuidad para la educación superior chilena", Claves de Políticas Públicas, núm. 7, pp. 1-14.

Bellei, C. (2015), El gran experimento: Mercado y privatización de la educación chilena, Santiago, LOM.

Beyer, H. y L. Cox (2011), "Gratuidad de la educación superior, una política regresiva", Puntos de Referencia, núm. 337, pp. 1-8.

Blanco, R. (2008), "Eficacia escolar desde el enfoque de calidad de la educación", en R. Blanco, I. Aguerrondo, G. Calvo, G. Cares, L. Cariola, R. Cervini y M. Zorrilla, Eficacia Escolar y factores asociados en America Latina y el Caribe, Santiago de Chile, OREALC/UnESCO, pp. 7-16.

Bravo, D. y J. Manzi (2002), "Equidad y resultados educacionales: SIMECE y PAA", Santiago,
Departamento de Economía Universidad de Chile/ Escuela de Psicología PUG.

Brunner, J. y D. Uribe (2007), Mercados universitarios: el nuevo escenario de la educación superior, Santiago, Ediciones Universidad Diego Portales.

Brunner, J. y G. Elacqua (2003), Informe capital humano en Chile, Santiago de Chile, Universidad Adolfo Ibáñez.

Castillo, J. y G. Cabezas (2010), "Caracterización de jóvenes primera generación en educación superior. Nuevas trayectorias hacia la equidad educativa", Calidad en la Educación, núm. 32, pp. 43-76.

Ciclo de Diálogos sobre el Futuro de la Educación (2014), Financiamiento de la educación en Chile: ¿Quién debe pagar?, Santiago de Chile, Centro de Estudios de Políticas y Prácticas en Educación CEPPE, Universidad Católica.

Contreras, M., F. Corbalán y J. Redondo (2007), "Cuando la suerte está hechada: estudio cuantitativo de los factores asociados al rendimiento en la PSU", Revista Iberoamericana sobre Calidad, Eficacia y Cambio en Educación, vol. 5, núm. 5, pp. 259-263.

Corti, A., D. Oliva y S. De la Cruz (2015), "La internacionalización y el mercado universitario", Revista de la Educación Superior, vol. 44, núm. 2, pp. 47-60. Corvalán, J. y J. García-Huidobro (2015), "La educación de mercado en Chile y su propuesta de superación", Cuadernos de educación, núm. 66, pp. 1-16. 
Corporación de Universidades Privadas de Chile (2016), Análisis de las minutas sobre proyecto de reforma a la educación superior, Santiago de Chile, Corporación de Universidades Privadas de Chile.

Dias, J. (2008), "Calidad, pertinencia y responsabilidad social de la universidad latinoamericana y caribeña", en A. Gazzola y A. Didriksson, Tendencias de la Educación Superior en América Latina y el Caribe, Caracas, IESALCUNESCO, pp. 87-112.

Donoso, S. (2002), "Los modelos de financiamiento de la educación pública en Chile y sus requerimientos de adecuación", Revista Electrónica de Investigación Educativa, vol. 4, núm. 2, pp. 151-172.

Donoso, S. y V. Cancino (2007), "Caracterización socioeconómica de los estudiantes de educación superior por tipo de institución", Calidad en la Educación, núm. 26, pp. 205-244.

Educación 2020 (2018), "Estos son 7 aspectos que debes saber sobre el lucro en la educación superior", <http:/ / educacion2020,cl/noticias/estos-son-7-aspectosque-debes-saber-sobre-el-lucro-en-la-educacionsuperior/> [Consulta: marzo de 2019].

Espinoza, O. (2013), Equidad e inclusividad en la educación superior en los países andinos: Los casos de Bolivia, Chile, Colombia y Perú, Santiago de Chile, Ediciones Universidad UCINF.

Espinoza, O. y L. González (2011), "Acceso a instituciones de educación superior públicas y privadas: el caso de Chile", en J. Brunner y C. Peña, El conflicto de las universidades: entre lo público y lo privado, Ediciones UDP, pp. 277-297.

Friedman, M. y R. Friedman (1980), Free to Choose, Orlando, Harcourt.

Gajardo, F. (2014), "Gratuidad en la educación superior: economía, política y evidencia”, Estudios Nueva Economía, vol. 3, núm. 1, pp. 56-66.

García, J. (2005), "La inserción laboral de los universitarios: sobrecualificación y desajuste formativo", España, IVIE y Universitat Pompeu Fabra, pp. 1-34.

García-Huidobro, J. y C. Bellei (2003), Desigualdad educativa en Chile, Santiago, Universidad Alberto Hurtado.
González, M. (2014), "Nuevas formas de gobernanza en la educación superior latinoamericana: Chile, Argentina y México", Bordón, Revista de Pedagogía, vol. 66, núm. 1, pp. 137-150.

Guerrero, L. y J. Campbell (2016), "Política de educacion superior en Chile: entre la calidad, equidad e innovación curricular", Revista Internacional de Educação Superior, vol. 2, núm. 1, pp. 51-66.

Herrera, J. (2013a), Economía de la educación y financiamiento de la educación superior, El ocaso del paradigma social: Universidad de Cartagena 1980-2002, Alemania, Publicia.

Herrera, J. (2013b), "Ética, equidad y meritocrácia en la mercantilización de la educación superior colombiana", Bioética, vol. 13, núm. 1, pp. 8-17.

Jiménez, A. (2009), "Reflexiones sobre la necesidad de acercamiento entre la universidad y el mercado laboral", Revista Iberoamerica de Educación, vol. 50, núm. 1, pp. 1-8.

Kremerman, M. (2013), El financiamiento a la educación en Chile: una mirada crítica, Puerto Montt, Escuela de Verano ACHM.

Larrañaga, O., G. Cabezas y F. Dussaillant (2013), Estudio de la Educación Técnico Profesional, Santiago de Chile, PNUD.

Ley 21091 (2018), Santiago, Biblioteca del Congreso Nacional de Chile, <https://www.leychile.cl/ Navegar?idNorma=1118991> [Consulta: mayo de 2019].

Ley Orgánica Constitucional de Educación (LOCE) (1990), Ley Orgánica Constitucional de Enseñanza (Núm. 18 962), Santiago, <http://www,uchile,cl/portal/ presentacion/normativa-y-reglamentos/8386/leyorganica-constitucional-de-ensenanza $>$ [Consulta: marzo de 2019].

Marcel, M. y C. Tokman (2005), ¿Cómo se financia la educación en Chile?, Santiago de Chile, Estudios de Finanzas Públicas.

Meller, P. y B. Lara (2010), Carreras universitarias: rentabilidad, selectividady discriminación, Santiago, Uqbar editores.

Mincer, J. (1958), "Investment in human capital and the personal income distribution", fournal of Political Economy, vol. 66, núm. 4, pp. 281-302. 
Organisation for Economic Cooperation and Development (OCDE) (2013), Panorama de la educación 2013, Indicadores de la OCDE, España, Santillana.

OCDE (2009), Revisión de políticas nacionales de educación: la educación superior en Chile, Santiago, OCDE y Banco Mundial.

Páez, A. y M. Kremerman (2016), Endeudar para gobernary mercantilizar: el caso del CAE, Santiago de Chile, Fundacion Sol.

Piscitelli, A., I. Adaime y I. Binder (2010), El proyecto Facebook y la posuniversidad: Sistemas operativos sociales y entornos abiertos de aprendizaje, Barcelona, Ariel.

Rodríguez, C. y G. Padilla (2017), "La privatización del financiamiento público en Chile: el subsidio a la oferta en el espacio universitario", Paradigma, vol. 38, núm. 2, pp. 27-47.

Rodríguez, E. (2012), "La educación superior en Chile y el rol del mercado: ¿culpable o inocente?", Ingeniare, Revista chilena de ingeniería, vol. 20, núm. 1, pp. 126-135.

Santa Cruz, E. y A. Olmedo (2012), "Neoliberalismo y creación de "sentido común": crisis educativa y medios de comunicación en Chile", Profesorado, vol. 16, núm. 3, pp. 145-168.

Smith, A. (1983), La riqueza de las naciones, España, Biblioteca de Economía 8, Orbis.
The World Bank (1988), Education in Sub-saharan Africa: policies for adjustment, revitalization, and expansion, Washington, D.G., The World Bank.

Urzúa, S. (2012), "La rentabilidad de la educación superior en Chile ¿educación superior para todos?”, Santiago, Centro de Estudios Públicos (Documento de Trabajo, 386), pp. 1-43.

Valdivieso, P. (2006), Caracterización educacional y sociodemográfica de los estudiantes que rinden la PSU, postulan y se matriculan en las universidades reunidas en el Consejo de Rectores, Santiago de Chile, Consejo de Educación Superior.

Valenzuela, J., C. Bellei y D. de Los Ríos (2010), "Segregación escolar en Chile", en Fin de ciclo, pp. 209229.

Vergara, R. (2007), “Tendencias demográficas y económicas en Chile y sus implicancias para la educación superior", Estudios Públicos, núm. 106, pp. 129-152.

Williamson, G. (2008), "El financiamiento a los estudiantes", en J. Brunner y C. Peña, Reforma de la educación superior, Santiago de Chile, Ediciones Universidad Diego Portales, pp. 121-148.

Zolezzi, J. y D. de los Ríos (2008), "El futuro de las universidades estatales", en J. Brunner y C. Peña, Reforma de la educación superior, Santiago de Chile, Universidad Diego Portales, pp. 377-402.

\section{Cómo citar este artículo:}

Rodríguez-Garcés, Carlos y Geraldo Padilla-Fuentes Pérez-Castro, Judith (2021), "Incorporación de la Ley de Gratuidad al Sistema de Educación Superior en Chile: la prolongación del subsidio a la demanda y la privatización del financiamiento público", Revista Iberoamericana de Educación Superior (RIES), vol. XII, núm. 33, pp. 179-195, DoI: https://doi.org/10.22201/iisue.20072872e.2021.33.864 [Consulta: fecha de última consulta]. 